\title{
Some Physical Properties of Spherical Amalgams for Commercial Use in the World
}

\author{
by \\ and Hiroyoshi $\mathrm{HABU}$ \\ Research Collaborators \\ 1. Introduction \\ 2. Experimental Methods and Materials \\ 3. Results \\ 3.1. Compressive strength \\ 3.2. Tensile strength \\ 3.3. Marginal strength \\ 3.4. Flow \\ 3.5. Hardness \\ 3.6. Dimensional change \\ 3.7. Corrosive tendency \\ 3.8. Initial hardening time of mix \\ 4. Discussion \\ 5. Conclusions \\ 6. References
}

Kazuo NAGAI, Masayoshi OHASHI

M. UEMURA, T. SHIMIZU, K. NEMOTO, K. HASEGAWA, T. MIYATA,

T. SHIMOJO, I. MAKI, Y. FUJIMOTO, F. YAMAKI and E. MEGURO

\section{Introduction}

One of the topics in the field of dental materials that have drawn a great amount of attention in recent years was undoubtedly the advent of spherical-particled amalgam a few years ago.

TAYLOR and his associates[1, 2, 3,] carried out a series of basic studies, from 1962 to 1967 , on the particle size and composition of this new amalgam alloy and gave their research findings that larger particles of amalgam alloy resulted in a large expansion at the time of setting, an increase in particle size decreased the residual mercury, particle size had no significant effect on flow, and particle sizes $15 \sim 30 \mu$ in diameter gave the maximum strength. WING and RYGE[4] studied the setting reaction of spherical amalgam by means of both the microscope and X-ray diffraction. According to KORAN and AsGaR[5], spherical amalgam packed under a smaller condensation pressure could have the same degree of the normal strength of conventional amalgam. In 1967, EDEN and WATERSTRAT[6] made it clear that spherical amalgam had a better

永井一夫, 大橋正敬, 土生博義, Dept. of Dental Materials, Nihon Univ. School of Dentistry, Tokyo, Japan. 
tensile strength as compared with conventional amalgam and that the former possessed a much advantageous fit.

The Shofu Dental Manufacturing Company, Kyoto, Japan, successfully produced spherical-particled amalgam alloy by a unique method and introduced it into the commercial market in 1965 for the first time in the world. NAGAI and his associates $[7,8,9,10,11$,$] undertook detailed studies of this new "Shofu Spherical Amalgam"$ that the effects of manipulative variables included its alloy-mercury ratio, condensation pressure, and condensing method on the properties such as compressive strength, dimensional change, hardness, flow, marginal strength, pre-setting flow and corrosive tendency, etc. On these findings, excellent properties of spherical amalgam have been confirmed in all respects. Because of the fact that spherical amalgam can be packed adequately under a small condensation pressure, it was suggested that this new amalgam was particularly useful in the pedodontic field[7]. As part of these research efforts, NAGAI and his associates [8] published an important recommendation concerning specification test on spherical amalgam.

In 1967 Plump[12], as a result of his 2-year observation of the lustre and marginal fit of spherical amalgam restorations and those of conventional amalgam, concluded that as compared with conventional, spherical amalgam gave much better clinical effects.

In 1966, Kerr, Co., U.S.A., produced a spherical amalgam alloy for commercial purposes. Currently in Japan, nearly $85 \%$ of 35,000 dental practitioners are estimated to be routine users of spherical amalgam and they are well satisfied with good clinical effects obtained.

The present study attempts comparative examinations of 5 currently available spherical amalgams (4 Japanese products and 1 U.S. product) in various properties to be described below.

\section{Experimental Methods and Materials}

As given in Table 1 and Fig. 1, 5 commercial spherical alloys were used. By way of control group, New True Dentalloy produced by S. S. White Co., a well-known conventional amalgam alloy was adopted. Fig. 2 shows photomicrographs of each alloy particles.

With $0.9 \mathrm{~g}$ alloy, mercury was measured out in compliance with the directions of these manufactures and subsequently triturated in the length of time as specified similarly. One deviation was made, however, in the case of Kerr Spheraloy. As the pellets of this product often failed to be properly crushed by a specified mixer of Toothmaster, it was replaced by G-C Mixer. Conventional amalgam was triturated in $1: 1$ alloy-mercury ratio and since there was no manufacturer's direction beyond $0.5 \mathrm{~g}$ alloy, the same procedure was repeated twice to obtain $1.0 \mathrm{~g}$ needed. To these standard triturations, effects of change in trituration time were added afterward.

An amalgam mix thus triturated was divided into 8 equal parts and condensed into a rigid steel die having a cavity $4 \mathrm{~mm}$ in diameter by the use of New "NON" condensing apparatus, having a plugger $3.9 \mathrm{~mm}$ in diameter under 80 thrusts by adding a twist movement (Fig. 3). It was already reported[10] that by the use of New "NON" 


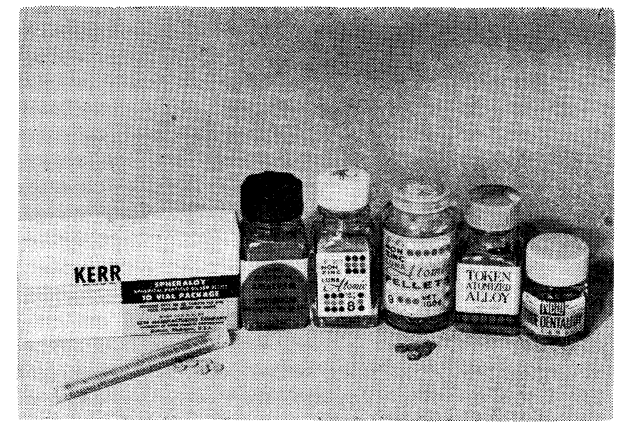

Fig. 1. Spherical alloys and a conventional alloy used in this study.

From left to right; Kerr Spheraloy, Shofu Spherical, G-C Atomic, G-C Atomic Pellet Toyo Atomized and S. S. White New True Dentalloy.

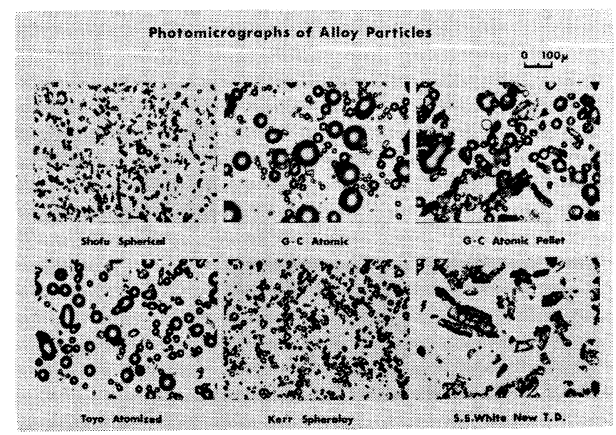

Fig. 2. Photomicrographs of alloy particles.



Fig. 3. A close-up of plugger point of New "NON" condensing apparatus having twist movement.

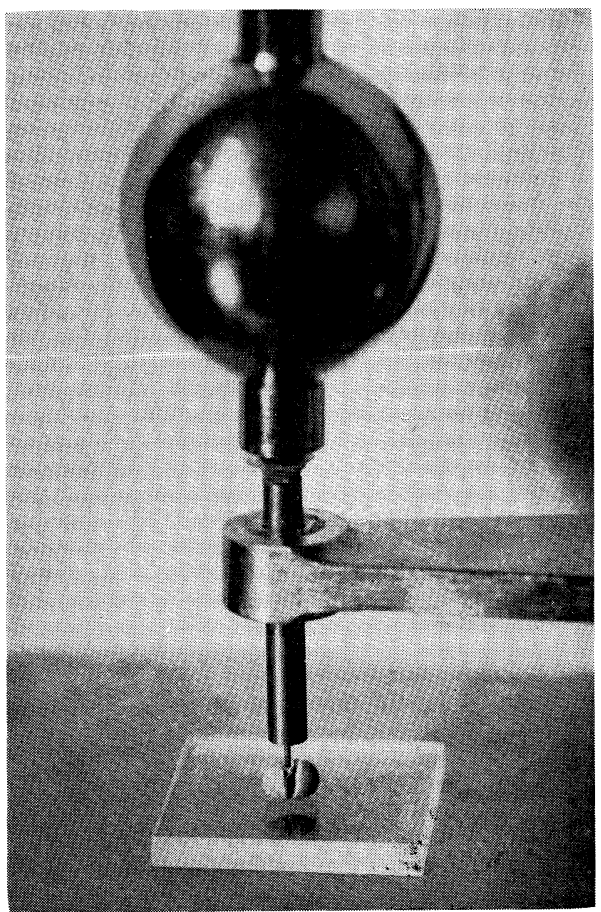

Fig. 4. Measurement of initial hardening time of amalgam mix ball. 
TABLE 1

Materials and Methods Studied

\begin{tabular}{|c|c|c|c|c|c|}
\hline Amalgam & Batch No. & Manufacturer & Alloy : $\mathrm{Hg}^{*}$ & Mixing* & Packing \\
\hline $\begin{array}{l}\text { Shofu } \\
\text { Spherical }\end{array}$ & 138 & $\begin{array}{l}\text { Shofu Dental } \\
\text { Mfg., Co. } \\
\text { Japan }\end{array}$ & $1.0: 0.87$ & $\begin{array}{l}\text { Shofu Mixer } \\
20 \text { sec. }\end{array}$ & $20 \mathrm{Kg} / \mathrm{cm}^{2}$ \\
\hline $\begin{array}{l}\text { Luna } \\
\text { Atomic }\end{array}$ & CL15 & $\begin{array}{l}\text { G-C Chemical } \\
\text { Mfg., Co. } \\
\text { Japan }\end{array}$ & $1.0: 0.86$ & $\begin{array}{l}\text { G-C Mixer } \\
\text { IIB } 5 \text { sec. }\end{array}$ & $20 \mathrm{Kg} / \mathrm{cm}^{2}$ \\
\hline $\begin{array}{l}\text { Luna } \\
\text { Atomic } \\
\text { Pellet }\end{array}$ & GL25 & $\begin{array}{l}\text { G-C Chemical } \\
\text { Mfg., Co. } \\
\text { Japan }\end{array}$ & $1.0: 0.9$ & $\begin{array}{l}\text { G-C Mixer } \\
\text { IIB } 8 \text { sec. }\end{array}$ & $20 \mathrm{Kg} / \mathrm{cm}^{2}$ \\
\hline $\begin{array}{l}\text { Toyo } \\
\text { Atomized }\end{array}$ & 121 & $\begin{array}{l}\text { Toyo Chemical } \\
\text { Lab. } \\
\text { Japan }\end{array}$ & $1.0: 1.0$ & $\begin{array}{l}\text { Shofu Mixer } \\
10 \text { sec. }\end{array}$ & $20 \mathrm{Kg} / \mathrm{cm}^{2}$ \\
\hline Spheraloy & $372 \mathrm{~K} 303$ & $\begin{array}{l}\text { Kerr Mfg., Co. } \\
\text { U.S.A. }\end{array}$ & $1.0: 1.0$ & $\begin{array}{l}\text { G-C Mixer } \\
\text { IIB } 20 \text { sec. }\end{array}$ & $20 \mathrm{Kg} / \mathrm{cm}^{2}$ \\
\hline $\begin{array}{l}\text { New True** } \\
\text { Dentalloy }\end{array}$ & 1246606 & $\begin{array}{l}\text { SS White Dental } \\
\text { Mfg., Co. } \\
\text { U.S.A. }\end{array}$ & $1.0: 1.0$ & $\begin{array}{l}\text { SS White } \\
\text { Mixer IIIA } \\
30 \text { sec. }\end{array}$ & $60 \mathrm{Kg} / \mathrm{cm}^{2}$ \\
\hline
\end{tabular}

mechanical condensing apparatus, individual variables could be checked at a minimum. According to our recent research[13], a condensation pressure of spherical amalgam while in the oral cavity was found to be unaffected by whether a plugger used was large or small in diameter, with about $20 \mathrm{Kg} / \mathrm{cm}^{2}$ more or less constantly measured. Therefore, for experimental purposes here spherical amalgam test specimen was prepared under $20 \mathrm{Kg} / \mathrm{cm}^{2}$, while conventional amalgam was condensed under $60 \mathrm{Kg} / \mathrm{cm}^{2}$ which is clinically concerned in maximum. Following the condensation a static load of uniform size was applied to the specimen for 1 minute, the entire operation taking 3 minutes 30 seconds since the start of trituration.

Upon removal of the final load, a sharp edge was applied to the top of a specimen and by trimming off about $3 \mathrm{~mm}$, a cylindrical specimen of $4 \mathrm{~mm}$ in diameter and $8 \mathrm{~mm}$ long was produced. Except for the measurements of marginal strength and initial hardening time, all the other tests were conducted on a test specimen of this dimension.

As for the measurement of tensile strength, use was made of diametral compression method on a cylindrical specimen[14].

A special method was designed, on the other hand, for the measurement of marginal strength, where an angle of $30^{\circ}$ was applied to a square test specimen of $2 \mathrm{~mm} \times 4 \mathrm{~mm} \times 15 \mathrm{~mm}$ and a length of fracture which occurred under a movement of a stainless steel ball $1 \mathrm{~mm}$ in diameter toward the margin with a load of $500 \mathrm{~g}$ was measured[8].

Respective corrosive tendencies of spherical and conventional amalgam specimens were electrochemically measured by means of the polarography $[11,15,16]$.

The initial hardening time of mix (the term setting time is generally used in this 
sense) was determined by applying loads of $\frac{1}{4}, \frac{1}{2}, 1$ and $2 \times 454 \mathrm{~g}$ (one pound) by the Gillmore needle with a flat end $1.06 \mathrm{~mm}$ in diameter on a ball of amalgam mix weighing about $1.8 \mathrm{~g}$. Measurement was made on this ball at an interval of every 5 seconds until the ball either did not break or had become hard enough to resist the penetration of needle (Fig. 4].

All the test specimens were prepared under room temperature of $23 \pm 2^{\circ} \mathrm{C}$ and, excepting for the balls of amalgam mix for measuring initial hardening time, they were immediately exposed to $37 \pm 1^{\circ} \mathrm{C}$. Tests on the flow and dimensional change were conducted at $37 \pm 1^{\circ} \mathrm{C}$ as prescribed by F. D. I. specification [17], whereas the remaining tests were conducted under $23 \pm 2^{\circ} \mathrm{C}$. In the results and discussion that follows, alloys will be abbreviated as; Shofu Spherical (S), G-C Atomic (GA), G-C Atomic Pellet (GP), Toyo Atomized (T), Kerr Spheraloy (K) and S. S. White New True Dentalloy (C).

\section{Results}

\subsection{Compressive strength}

The compressive strength of test specimens was measured by use of Amsler Universal Tester under a head speed of $1 \mathrm{~mm}$ per minute. Means of 5 measurements are given in Table 2 and Fig. 5.

As is known, $(\mathrm{S}),(\mathrm{T})$, and $(\mathrm{K})$ gave more or less the same tendency. They registered a high initial strength and an increase in the strength was seen at 5 hours. After 24 hours, a significant difference was computed between these three and other spherical alloy specimens inclusive those of conventional amalgam. Although (GA)

TABLE 2

Comparison of Compressive Strengths

\begin{tabular}{|c|c|c|c|c|c|c|c|}
\hline \multirow{3}{*}{ Amalgam } & \multirow{3}{*}{$\begin{array}{c}\text { Time of } \\
\text { Mix } \\
\text { (sec.) }\end{array}$} & \multicolumn{5}{|c|}{ Age of Specimen (Hours) } & \multirow{3}{*}{$\begin{array}{c}\text { S.D. } \\
\text { at 24hrs }\end{array}$} \\
\hline & & 1 & 3 & 5 & 10 & 24 & \\
\hline & & \multicolumn{5}{|c|}{ Compressive Strength $\left(\mathrm{Kg} / \mathrm{cm}^{2}\right)$. } & \\
\hline $\begin{array}{l}\text { Shofu } \\
\text { Spherical }\end{array}$ & $\begin{array}{l}15 \\
20 *\end{array}$ & $\begin{array}{l}1,663 \\
1,822\end{array}$ & $\begin{array}{l}2,609 \\
2,920\end{array}$ & $\begin{array}{l}3,484 \\
3,707\end{array}$ & $\begin{array}{l}3,811 \\
3,938\end{array}$ & $\begin{array}{l}3,938 \\
4,115\end{array}$ & $\begin{array}{l}94 \\
94\end{array}$ \\
\hline $\begin{array}{l}\text { G-C } \\
\text { Atomic }\end{array}$ & $\begin{array}{l}5^{*} \\
10\end{array}$ & $\begin{array}{l}1,456 \\
1,591\end{array}$ & $\begin{array}{l}2,148 \\
2,196\end{array}$ & $\begin{array}{l}2,514 \\
2,609\end{array}$ & $\begin{array}{l}3,246 \\
3,405\end{array}$ & $\begin{array}{l}3,532 \\
3,644\end{array}$ & $\begin{array}{l}81 \\
58\end{array}$ \\
\hline $\begin{array}{l}\text { G-C } \\
\text { Atomic Pellet }\end{array}$ & $\begin{array}{l}8^{*} \\
15\end{array}$ & $\begin{array}{l}1,098 \\
1,090\end{array}$ & $\begin{array}{l}2,331 \\
2,777\end{array}$ & $\begin{array}{l}3,126 \\
3,294\end{array}$ & $\begin{array}{l}3,500 \\
3,516\end{array}$ & $\begin{array}{l}3,572 \\
3,683\end{array}$ & $\begin{array}{r}85 \\
165\end{array}$ \\
\hline $\begin{array}{l}\text { Toyo } \\
\text { Atomized }\end{array}$ & $\begin{array}{l}10 \\
20^{*}\end{array}$ & $\begin{array}{l}1,531 \\
1,687\end{array}$ & $\begin{array}{l}2,495 \\
2,625\end{array}$ & $\begin{array}{l}3,348 \\
3,433\end{array}$ & $\begin{array}{l}3,675 \\
3,787\end{array}$ & $\begin{array}{l}3,967 \\
4,077\end{array}$ & $\begin{array}{l}82 \\
52\end{array}$ \\
\hline $\begin{array}{l}\text { Kerr } \\
\text { Spheraloy }\end{array}$ & $\begin{array}{l}20^{*} \\
30\end{array}$ & $\begin{array}{l}1,575 \\
1,599\end{array}$ & $\begin{array}{l}3,341 \\
2,469\end{array}$ & $\begin{array}{l}3,477 \\
3,612\end{array}$ & $\begin{array}{l}3,962 \\
4,026\end{array}$ & $\begin{array}{l}4,163 \\
4,306\end{array}$ & $\begin{array}{l}185 \\
142\end{array}$ \\
\hline $\begin{array}{l}\text { SS. White** } \\
\text { New T. D. }\end{array}$ & $30^{*}$ & 1,308 & 2,681 & 3,122 & 3,421 & 3,594 & 95 \\
\hline
\end{tabular}

* Manufacturer's direction.

** Conventional alloy as control. 


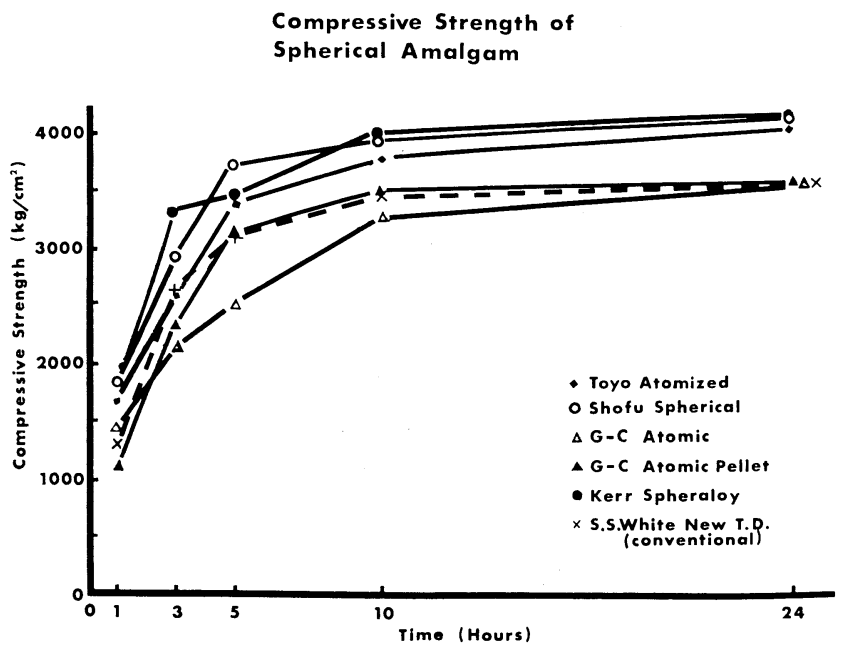

Fig. 5

gave a little higher strength than conventional amalgam (C) after one hour, it turned to become much reduced than (C) in the measurements of 3 and 5 hours respectively. (GP), having the usual tendency of conventional amalgam, was found to be in the middle. In general the compressive strength increases proportionally to the duration of trituration effected but change here is by no means large.

\subsection{Tensile strength}

Means of 5 measurements as undertaken by the diametral compression method are given in Table 3 and Fig. 6. The tensile strength gave similar result as that of the compressive strength and, in this connection, (GA) gave much reduced value in the measurements at 3 and 5 hours. After 24 hours, the order of tensile strengths was $(\mathrm{K}),(\mathrm{S}),(\mathrm{C}),(\mathrm{T}),(\mathrm{GA})$ and $(\mathrm{GP})$.

TABLE 3

Comparison of Tensile Strengths

\begin{tabular}{|c|c|c|c|c|c|c|c|}
\hline \multirow{3}{*}{ Amalgam } & \multirow{3}{*}{$\begin{array}{l}\text { Time of } \\
\text { Mix } \\
\text { (Sec.) }\end{array}$} & \multicolumn{5}{|c|}{ Age of Specimen (Hours) } & \multirow{3}{*}{$\begin{array}{l}\text { S.D. } \\
\text { at 24hrs }\end{array}$} \\
\hline & & 1 & 3 & 5 & 10 & 24 & \\
\hline & & \multicolumn{5}{|c|}{ Tensile Strength $\left(\mathrm{Kg} / \mathrm{cm}^{2}\right)$} & \\
\hline $\begin{array}{l}\text { Shofu } \\
\text { Spherical }\end{array}$ & $\begin{array}{l}15 \\
20^{*}\end{array}$ & $\begin{array}{l}214 \\
221\end{array}$ & $\begin{array}{l}338 \\
358\end{array}$ & $\begin{array}{l}547 \\
566\end{array}$ & $\begin{array}{l}643 \\
667\end{array}$ & $\begin{array}{l}647 \\
676\end{array}$ & $\begin{array}{l}38 \\
23\end{array}$ \\
\hline $\begin{array}{l}\text { G-C } \\
\text { Atomic }\end{array}$ & $\begin{array}{c}5^{*} \\
10\end{array}$ & $\begin{array}{l}155 \\
202\end{array}$ & $\begin{array}{l}228 \\
267\end{array}$ & $\begin{array}{l}276 \\
287\end{array}$ & $\begin{array}{l}532 \\
566\end{array}$ & $\begin{array}{l}605 \\
620\end{array}$ & $\begin{array}{l}11 \\
16\end{array}$ \\
\hline $\begin{array}{l}\text { G-C } \\
\text { Atomic Pellet }\end{array}$ & $\stackrel{8^{*}}{15}$ & $\begin{array}{l}166 \\
171\end{array}$ & $\begin{array}{l}303 \\
311\end{array}$ & $\begin{array}{l}471 \\
515\end{array}$ & $\begin{array}{l}554 \\
568\end{array}$ & $\begin{array}{l}590 \\
606\end{array}$ & $\begin{array}{l}32 \\
30\end{array}$ \\
\hline $\begin{array}{l}\text { Toyo } \\
\text { Atomized }\end{array}$ & $\begin{array}{l}10 \\
20^{*}\end{array}$ & $\begin{array}{l}232 \\
246\end{array}$ & $\begin{array}{l}339 \\
358\end{array}$ & $\begin{array}{l}450 \\
505\end{array}$ & $\begin{array}{l}524 \\
552\end{array}$ & $\begin{array}{l}556 \\
635\end{array}$ & $\begin{array}{l}17 \\
17\end{array}$ \\
\hline $\begin{array}{l}\text { Kerr } \\
\text { Spheraloy }\end{array}$ & $\begin{array}{l}20 * \\
30\end{array}$ & $\begin{array}{l}208 \\
222\end{array}$ & $\begin{array}{l}384 \\
418\end{array}$ & $\begin{array}{l}561 \\
591\end{array}$ & $\begin{array}{l}620 \\
682\end{array}$ & $\begin{array}{l}709 \\
724\end{array}$ & $\begin{array}{l}18 \\
15\end{array}$ \\
\hline $\begin{array}{l}\text { SS White** } \\
\text { New T.D. }\end{array}$ & $30^{*}$ & 158 & 340 & 535 & 622 & 640 & 16 \\
\hline
\end{tabular}

* Manufacturer's direction.

** Conventional alloy as control. 


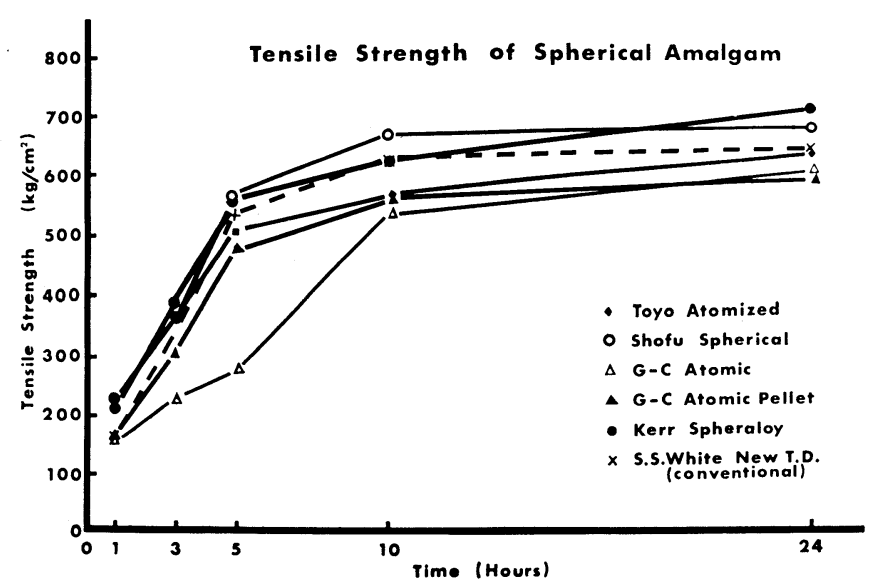

Fig. 6

\subsection{Marginal strength}

Table 4 and Fig. 7 are concerned with the marginal strength of amalgams having an angle of $30^{\circ}$. The longer is a length of fracture caused, the weaker is the marginal strength. After 5 hours, (GA) was found to be the weakest in its margin of all the rest. This tendency also continued to the passage of time.

TABLE 4

Comparison of Marginal Strengths

\begin{tabular}{|c|c|c|c|c|c|c|}
\hline \multirow{3}{*}{ Amalgam } & \multirow{3}{*}{$\begin{array}{l}\text { Time of } \\
\text { Mix } \\
\text { (Sec.) }\end{array}$} & \multicolumn{4}{|c|}{ Age of Specimen } & \multirow{3}{*}{$\begin{array}{c}\text { S.D. } \\
\text { at } 7 \text { days }\end{array}$} \\
\hline & & $5 \mathrm{hrs}$ & $10 \mathrm{hrs}$ & $24 \mathrm{hrs}$ & 7 days & \\
\hline & & \multicolumn{4}{|c|}{ Length of Fracture $(\mu)$} & \\
\hline $\begin{array}{l}\text { Shofu } \\
\text { Spherical }\end{array}$ & $\begin{array}{l}15 \\
20^{*}\end{array}$ & $\begin{array}{l}530 \\
533\end{array}$ & $\begin{array}{l}380 \\
387\end{array}$ & $\begin{array}{l}380 \\
386\end{array}$ & $\begin{array}{l}368 \\
369\end{array}$ & $\begin{array}{r}16 \\
8\end{array}$ \\
\hline $\begin{array}{l}\text { G-C } \\
\text { Atomic }\end{array}$ & $\begin{array}{l}5^{*} \\
10\end{array}$ & $\begin{array}{l}731 \\
630\end{array}$ & $\begin{array}{l}488 \\
480\end{array}$ & $\begin{array}{l}441 \\
417\end{array}$ & $\begin{array}{l}434 \\
401\end{array}$ & $\begin{array}{l}16 \\
17\end{array}$ \\
\hline $\begin{array}{l}\text { G-C } \\
\text { Atomic Pellet }\end{array}$ & $\begin{array}{l}8^{*} \\
15\end{array}$ & $\begin{array}{l}551 \\
536\end{array}$ & $\begin{array}{l}464 \\
425\end{array}$ & $\begin{array}{l}420 \\
407\end{array}$ & $\begin{array}{l}399 \\
395\end{array}$ & $\begin{array}{l}26 \\
13\end{array}$ \\
\hline $\begin{array}{l}\text { Toyo } \\
\text { Atomized }\end{array}$ & $\begin{array}{l}10 \\
20 *\end{array}$ & $\begin{array}{l}507 \\
501\end{array}$ & $\begin{array}{l}413 \\
410\end{array}$ & $\begin{array}{l}402 \\
404\end{array}$ & $\begin{array}{l}398 \\
384\end{array}$ & $\begin{array}{l}23 \\
15\end{array}$ \\
\hline $\begin{array}{l}\text { Kerr } \\
\text { Spheraloy }\end{array}$ & $\begin{array}{l}20^{*} \\
30\end{array}$ & $\begin{array}{l}504 \\
510\end{array}$ & $\begin{array}{l}390 \\
411\end{array}$ & $\begin{array}{l}389 \\
389\end{array}$ & $\begin{array}{l}380 \\
381\end{array}$ & $\begin{array}{l}34 \\
30\end{array}$ \\
\hline $\begin{array}{l}\text { SS White** } \\
\text { New T.D. }\end{array}$ & $30^{*}$ & 551 & 473 & 402 & 390 & 24 \\
\hline
\end{tabular}

* Manufacturer's direction.

** Conventional alloy as control.

Table 5 and Fig. 8 are comparison of 3 different strengths with the value of (C) as 100 , at 5 hour age. 
TABLE 5

Comparison of Three Different Strengths at 5-Hour Age

\begin{tabular}{|c|c|c|c|}
\hline \multirow[t]{2}{*}{ Amalgam } & Compressive & $\begin{array}{l}\text { Strength } \\
\text { Tensile }\end{array}$ & Marginal \\
\hline & \multicolumn{3}{|c|}{ Per Cent* } \\
\hline $\begin{array}{l}\text { Shofu } \\
\text { Spherical }\end{array}$ & 118.7 & 105.8 & 103.4 \\
\hline $\begin{array}{l}\text { G-C } \\
\text { Atomic }\end{array}$ & 80.5 & 51.6 & 75.4 \\
\hline $\begin{array}{l}\text { G-C } \\
\text { Atomic Pellet }\end{array}$ & 100.1 & 88.0 & 100.0 \\
\hline $\begin{array}{l}\text { Toyo } \\
\text { Atomized }\end{array}$ & 110.0 & 94.4 & 110.0 \\
\hline $\begin{array}{l}\text { Kerr } \\
\text { Spheraloy }\end{array}$ & 111.4 & 104.9 & 109.3 \\
\hline $\begin{array}{l}\text { SS White** } \\
\text { New T.D. }\end{array}$ & 100.0 & 100.0 & 100.0 \\
\hline
\end{tabular}

* Figures are based on the values of conventional alloy as 100 .

** Conventional alloy as control.

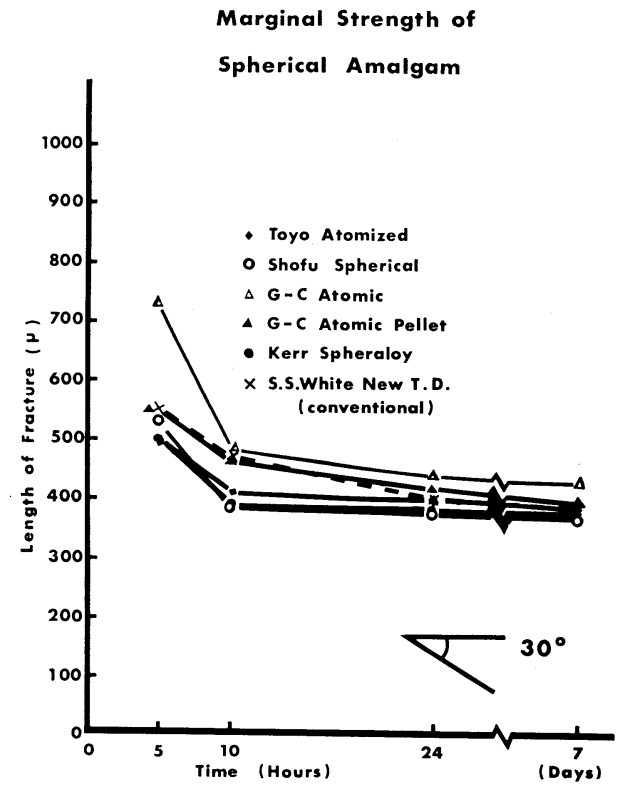

Fig. 7

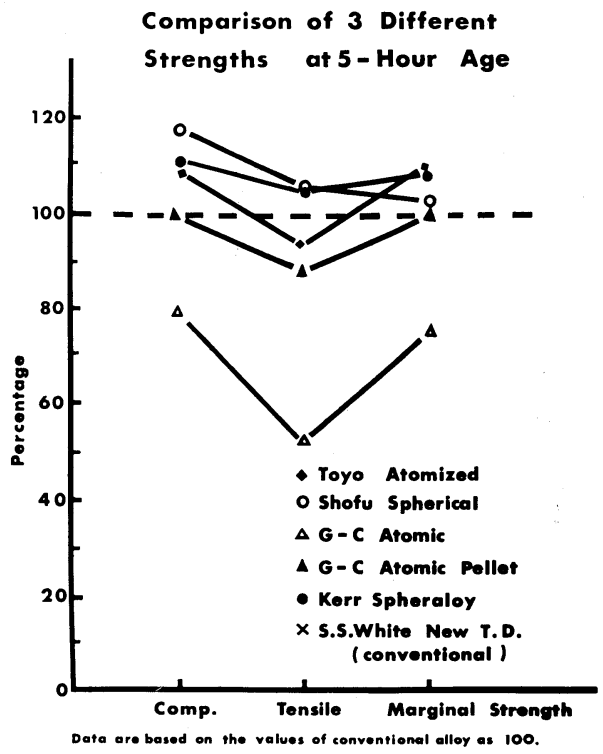

Fig. 8 


\subsection{Flow}

Table 6 and Fig. 9 are means of 5 measurements of flow by modfied Micro Brinell Hardness Tester with the precision of $1 \mu$. Order of the flow (from small to large) was (S) with $0.5 \%$, (T), (C), (GP), (GA) and (K) with $1.2 \%$, after 24 hours. Despite a satisfactory compressive strength of $(\mathrm{K})$, its flow was unexpectedly large, though being within the range prescribed in F.D.I. specification.

Influence of the trituration time, as has been known from the above, made itself felt more on the flow rather than on the strengths of an amalgam. While the flow of alloys (S) and (T) were not appreciably influenced by the duration of trituration, (GA) and (GP) were correspondingly influenced proportionally to the length of trituration.

TABLE 6

Comparison of Flows

\begin{tabular}{|c|c|c|c|c|c|c|c|}
\hline \multirow{3}{*}{ Amalgam } & \multirow{3}{*}{$\begin{array}{c}\text { Time of } \\
\text { Mix (Sec.) }\end{array}$} & \multicolumn{5}{|c|}{ Age of Specimen (Hours) } & \multirow{3}{*}{$\begin{array}{c}\text { S.D. } \\
\text { at } 24 \mathrm{hrs} \text {. }\end{array}$} \\
\hline & & 4 & 5 & 6 & 12 & 24 & \\
\hline & & \multicolumn{5}{|c|}{ Flow $(\%)$} & \\
\hline $\begin{array}{l}\text { Shofu } \\
\text { Spherical }\end{array}$ & $\begin{array}{l}15 \\
20^{*}\end{array}$ & $\begin{array}{l}0.26 \\
0.28\end{array}$ & $\begin{array}{l}0.31 \\
0.32\end{array}$ & $\begin{array}{l}0.33 \\
0.35\end{array}$ & $\begin{array}{l}0.38 \\
0.40\end{array}$ & $\begin{array}{l}0.42 \\
0.46\end{array}$ & $\begin{array}{l}0.06 \\
0.04\end{array}$ \\
\hline $\begin{array}{l}\text { G-C } \\
\text { Atomic }\end{array}$ & $\begin{array}{l}5^{*} \\
10\end{array}$ & $\begin{array}{l}0.48 \\
0.68\end{array}$ & $\begin{array}{l}0.59 \\
0.85\end{array}$ & $\begin{array}{l}0.68 \\
0.97\end{array}$ & $\begin{array}{l}0.93 \\
1.36\end{array}$ & $\begin{array}{l}1.14 \\
1.67\end{array}$ & $\begin{array}{l}0.10 \\
0.19\end{array}$ \\
\hline $\begin{array}{l}\text { G-C } \\
\text { Atomic Pellet }\end{array}$ & $\begin{array}{l}8^{*} \\
15\end{array}$ & $\begin{array}{l}0.54 \\
0.75\end{array}$ & $\begin{array}{l}0.63 \\
0.94\end{array}$ & $\begin{array}{l}0.68 \\
1.06\end{array}$ & $\begin{array}{l}0.84 \\
1,35\end{array}$ & $\begin{array}{l}1.03 \\
1.69\end{array}$ & $\begin{array}{l}0.14 \\
0.22\end{array}$ \\
\hline $\begin{array}{l}\text { Toyo } \\
\text { Atomized }\end{array}$ & $\begin{array}{l}10 \\
20^{*}\end{array}$ & $\begin{array}{l}0.39 \\
0.41\end{array}$ & $\begin{array}{l}0.44 \\
0.46\end{array}$ & $\begin{array}{l}0.47 \\
0.48\end{array}$ & $\begin{array}{l}0.55 \\
0.56\end{array}$ & $\begin{array}{l}0.61 \\
0.63\end{array}$ & $\begin{array}{l}0.07 \\
0.08\end{array}$ \\
\hline $\begin{array}{l}\text { Kerr } \\
\text { Spheraloy }\end{array}$ & $\begin{array}{l}20^{*} \\
30\end{array}$ & $\begin{array}{l}0.67 \\
0.82\end{array}$ & $\begin{array}{l}0.85 \\
1.07\end{array}$ & $\begin{array}{l}0.93 \\
1.16\end{array}$ & $\begin{array}{l}1.09 \\
1.33\end{array}$ & $\begin{array}{l}1.23 \\
1.47\end{array}$ & $\begin{array}{l}0.12 \\
0.11\end{array}$ \\
\hline $\begin{array}{l}\text { SS White** } \\
\text { New T. D. }\end{array}$ & $30^{*}$ & 0.43 & 0.49 & 0.53 & 0.64 & 0.76 & 0.08 \\
\hline
\end{tabular}

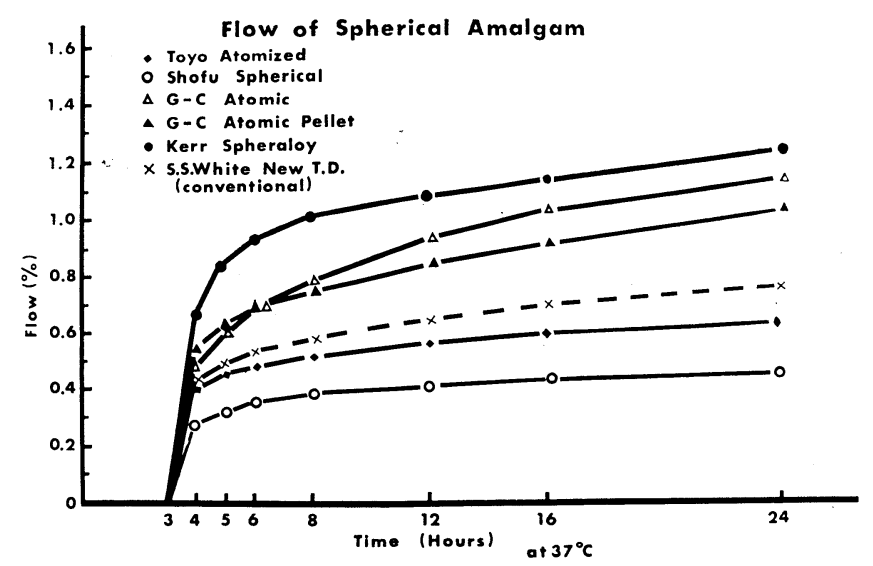

Fig. 9 


\subsection{Hardness}

Means of Vickers hardness measured on 5 different spots on 2 similarly prepared specimens are given in Table 7 and Fig. 10. After 24 hours, alloy (T) registered the highest hardness of all the rest.

TABLE 7

Comparison of Vickers Hardnesses

\begin{tabular}{|c|c|c|c|c|c|c|c|}
\hline \multirow{3}{*}{ Amalgam } & \multirow{3}{*}{$\begin{array}{c}\text { Time of } \\
\text { Mix (Sec.) }\end{array}$} & \multicolumn{5}{|c|}{ Age of Specimen (Hours) } & \multirow{3}{*}{$\begin{array}{l}\text { S.D. } \\
\text { at } 24 \mathrm{hrs} \text {. }\end{array}$} \\
\hline & & 1 & 3 & 5 & 7 & 24 & \\
\hline & & \multicolumn{5}{|c|}{ Vickers Hardness Number } & \\
\hline $\begin{array}{l}\text { Shofu } \\
\text { Spherical }\end{array}$ & $\begin{array}{l}15 \\
20 *\end{array}$ & $\begin{array}{l}61.7 \\
57.4\end{array}$ & $\begin{array}{l}84.2 \\
87.3\end{array}$ & $\begin{array}{r}97.6 \\
107.1\end{array}$ & $\begin{array}{l}117.9 \\
116.9\end{array}$ & $\begin{array}{l}119.0 \\
134.2\end{array}$ & $\begin{array}{l}11.9 \\
14.5\end{array}$ \\
\hline $\begin{array}{l}\text { G-C } \\
\text { Atomic }\end{array}$ & $\begin{array}{c}5^{*} \\
10\end{array}$ & $\begin{array}{l}48.3 \\
61.0\end{array}$ & $\begin{array}{l}95.3 \\
98.4\end{array}$ & $\begin{array}{r}95.6 \\
111.8\end{array}$ & $\begin{array}{l}102.1 \\
113.4\end{array}$ & $\begin{array}{l}111.8 \\
123.1\end{array}$ & $\begin{array}{l}11.4 \\
22.3\end{array}$ \\
\hline $\begin{array}{l}\text { G-C } \\
\text { Atomic Pellet }\end{array}$ & $\begin{array}{c}8^{*} \\
15\end{array}$ & $\begin{array}{l}67.1 \\
64.3\end{array}$ & $\begin{array}{l}82.8 \\
82.3\end{array}$ & $\begin{array}{l}94.2 \\
97.8\end{array}$ & $\begin{array}{l}109.0 \\
116.1\end{array}$ & $\begin{array}{l}118.4 \\
121.6\end{array}$ & $\begin{array}{l}20.2 \\
19.8\end{array}$ \\
\hline $\begin{array}{l}\text { Toyo } \\
\text { Atomized }\end{array}$ & $\begin{array}{l}10 \\
20^{*}\end{array}$ & $\begin{array}{l}78.3 \\
63.0\end{array}$ & $\begin{array}{l}98.0 \\
85.9\end{array}$ & $\begin{array}{r}117.9 \\
98.6\end{array}$ & $\begin{array}{l}122.2 \\
109.9\end{array}$ & $\begin{array}{l}135.4 \\
141.7\end{array}$ & $\begin{array}{l}24.0 \\
11.1\end{array}$ \\
\hline $\begin{array}{l}\text { Kerr } \\
\text { Spheraloy }\end{array}$ & $\begin{array}{l}20 * \\
30\end{array}$ & $\begin{array}{l}58.9 \\
57.4\end{array}$ & $\begin{array}{l}86.8 \\
80.1\end{array}$ & $\begin{array}{r}103.8 \\
99.0\end{array}$ & $\begin{array}{l}114.8 \\
106.6\end{array}$ & $\begin{array}{l}116.0 \\
127.5\end{array}$ & $\begin{array}{l}14.5 \\
11.7\end{array}$ \\
\hline $\begin{array}{l}\text { SS White** } \\
\text { New T.D. }\end{array}$ & $30^{*}$ & 47.4 & 73.5 & 93.2 & 94.0 & 113.3 & 17.6 \\
\hline
\end{tabular}

* Manufacturer's direction.

** Conventional alloy as control.

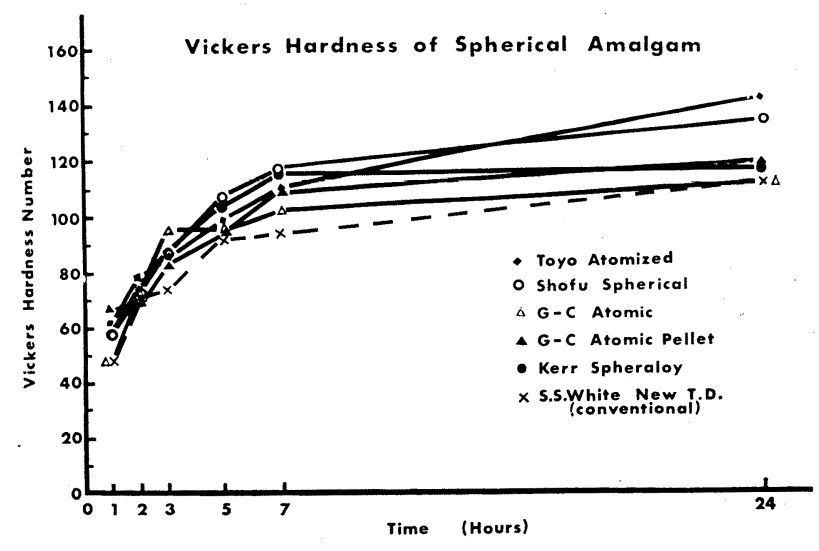

Fig. 10

\subsection{Dimensional change}

Table 8 and Fig. 11 relate to means of 5 measurements of dimensional change, in which an electrical micrometer with the precision of $0.5 \mu$. Two G-C products gave an expansion of about $12 \mu / \mathrm{cm}$ after 24 hours, while the rest of amalgams registered shrinkage on the contrary. 
TABLE 8

Comparison of Dimensional Changes

\begin{tabular}{|c|c|c|c|c|c|c|c|}
\hline \multirow{3}{*}{ Amalgam } & \multirow{3}{*}{$\begin{array}{l}\text { Time of* } \\
\text { Mix (Sec.) }\end{array}$} & \multicolumn{5}{|c|}{ Age of Specimen (Hours) } & \multirow{3}{*}{$\begin{array}{l}\text { S.D. } \\
\text { at } 24 \mathrm{hrs}\end{array}$} \\
\hline & & 1 & 2 & 3 & 12 & 24 & \\
\hline & & \multicolumn{5}{|c|}{ Dimensional Change $(\mu / \mathrm{cm})$} & \\
\hline $\begin{array}{l}\text { Shofu } \\
\text { Spherical }\end{array}$ & 20 & +0.9 & 0 & -0.6 & -1.8 & -2.4 & 0.3 \\
\hline $\begin{array}{l}\text { G-C } \\
\text { Atomic }\end{array}$ & 5 & +3.3 & +5.1 & +6.4 & +12.0 & +12.0 & 0.3 \\
\hline $\begin{array}{l}\text { G-C } \\
\text { Atomic Pellet }\end{array}$ & 8 & +7.1 & +9.9 & +10.9 & +11.6 & +11.6 & 0.8 \\
\hline $\begin{array}{l}\text { Toyo } \\
\text { Atomized }\end{array}$ & 20 & +1.2 & +0.9 & +0.6 & -0.3 & -0.6 & 0.3 \\
\hline $\begin{array}{l}\text { Kerr } \\
\text { Spheraloy }\end{array}$ & 20 & -1.9 & -4.8 & -7.4 & -13.2 & -13.9 & 0.9 \\
\hline $\begin{array}{l}\text { SS White** } \\
\text { New T. D. }\end{array}$ & 30 & 0 & +1.0 & +1.1 & -0.5 & -0.8 & 0.3 \\
\hline
\end{tabular}

* Manufacturer's direction.

** Conventional alloy as control.

Dimensional Change of Spherical Amalgam

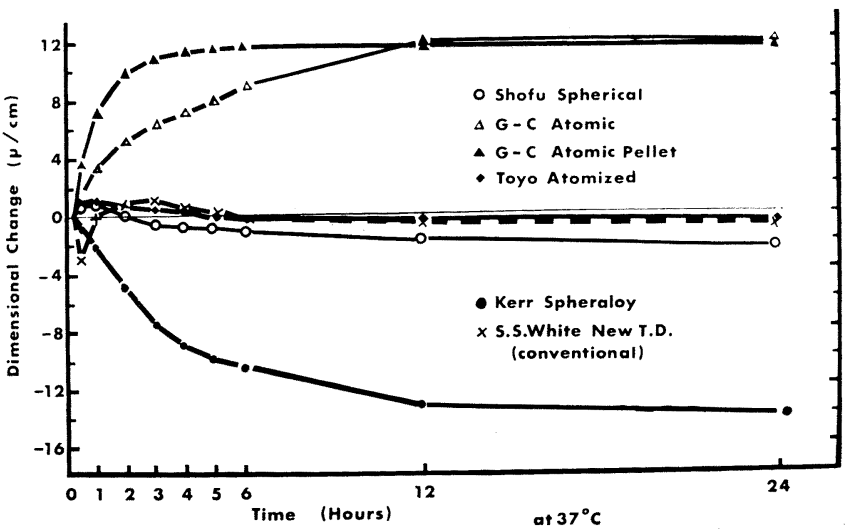

Fig. 11

When the trituration was effected longer than was indicated in a manufacturer's direction, shrinkage increased or expansion reduced itself. On the other hand, when the trituration was shorter than was indicated, expansion increased or inversely, shrinkage decreased. (S) and (T) shrank a little using manufacturer's directions while they expanded when triturated for a short duration of 5 seconds as direction of (GA). (Table 9 and Fig. 12). 


\section{TABLE 9}

Effect of Mixing Time on Dimensional Changes at 24-Hour Age

\begin{tabular}{|c|c|c|c|c|c|c|}
\hline \multirow{3}{*}{ Amalgam } & \multicolumn{6}{|c|}{ Time of Mix (Sec.) } \\
\hline & 5 & 8 & 10 & 15 & 20 & 30 \\
\hline & \multicolumn{6}{|c|}{ Dimensional Change $(\mu / \mathrm{cm})$} \\
\hline $\begin{array}{l}\text { Shofu } \\
\text { Spherical }\end{array}$ & +1.4 & & & -0.9 & $-2.4^{*}$ & \\
\hline $\begin{array}{l}\text { G-C } \\
\text { Atomic }\end{array}$ & $+12.0 *$ & & +9.8 & & +4.3 & \\
\hline $\begin{array}{l}\text { G-C } \\
\text { Atomic Pellet }\end{array}$ & & $+11.6^{*}$ & & +6.8 & +4.2 & \\
\hline $\begin{array}{l}\text { Toyo } \\
\text { Atomized }\end{array}$ & +2.6 & & +1.2 & & $-0.6^{*}$ & \\
\hline $\begin{array}{l}\text { Kerr } \\
\text { Spheraloy }\end{array}$ & & & -10.6 & & $-13.9^{*}$ & -17.3 \\
\hline $\begin{array}{l}\text { SS White** } \\
\text { New T.D. }\end{array}$ & & & & & & $-0.8^{*}$ \\
\hline
\end{tabular}

Effect of Mixing Time on Dimensional

Changes at 24 Hour Age

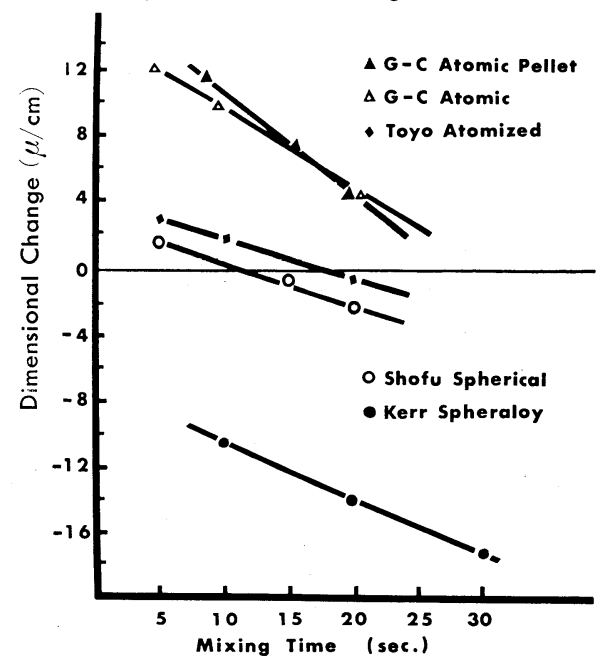

Fig. 12

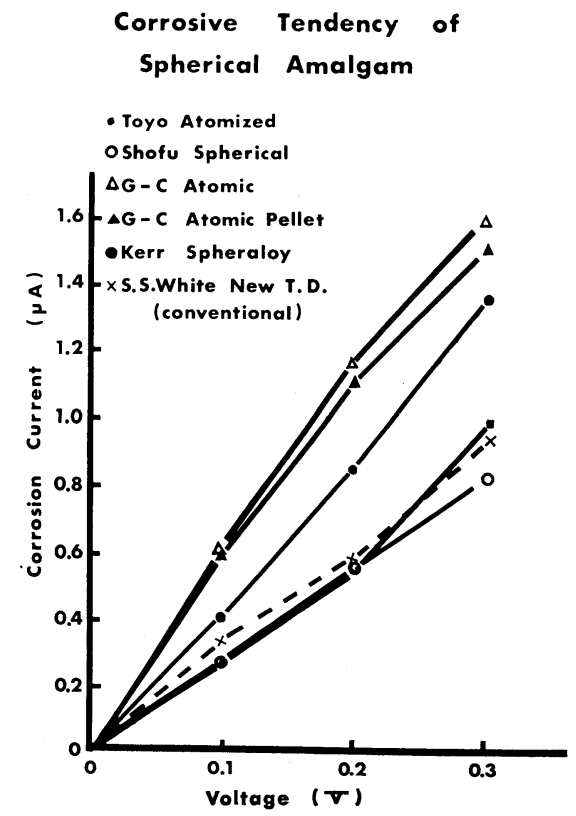

Fig. 13 


\subsection{Corrosive tendency}

Polarographic findings of 6 amalgam alloys are given in Table 10 and Fig. 13, where the corrosive agent was artificial saliva. Less electric current is interpreted as having a better corrosive resistance. By this polarographic method, it has been previously demonstrated that we could electrochemically measure the corrosive tendency of an alloy quickly and accurately in the state approximating the oral cavity.[11, 15, 16].

$(\mathrm{S}),(\mathrm{T})$ and $(\mathrm{C})$ proved themselves more corrosive resistant, $(\mathrm{K})$ was a little less than conventional alloy and two G-C products gave the least resistance to corrosion.

TABLE 10

Corrosive Values at Three Different Voltages

\begin{tabular}{|c|c|c|c|c|c|}
\hline \multirow[t]{2}{*}{ Amalgam } & \multirow[t]{2}{*}{$\begin{array}{l}\text { Time of Mix } \\
\quad \text { (Sec.) }\end{array}$} & $0.1 \mathrm{~V}$ & $\begin{array}{c}\text { At } \\
0.2 \mathrm{~V}\end{array}$ & $0.3 \mathrm{~V}$ & \multirow{2}{*}{$\begin{array}{l}\text { S.D. at } \\
0.2 \mathrm{~V}\end{array}$} \\
\hline & & \multicolumn{3}{|c|}{ Corrosion Current $(\mu \mathrm{A})$} & \\
\hline $\begin{array}{l}\text { Shofu } \\
\text { Spherical }\end{array}$ & $\begin{array}{l}15 \\
20^{*}\end{array}$ & $\begin{array}{l}0.30 \\
0.28\end{array}$ & $\begin{array}{l}0.59 \\
0.55\end{array}$ & $\begin{array}{l}0.93 \\
0.82\end{array}$ & $\begin{array}{l}0.06 \\
0.06\end{array}$ \\
\hline $\begin{array}{l}\text { G-C } \\
\text { Atomic }\end{array}$ & $\begin{array}{c}5^{*} \\
10\end{array}$ & $\begin{array}{l}0.61 \\
0.41\end{array}$ & $\begin{array}{l}1.16 \\
0.78\end{array}$ & $\begin{array}{l}1.59 \\
1.24\end{array}$ & $\begin{array}{l}0.08 \\
0.11\end{array}$ \\
\hline $\begin{array}{l}\text { G-C } \\
\text { Atomic Pellet }\end{array}$ & $\begin{array}{c}8^{*} \\
15\end{array}$ & $\begin{array}{l}0.60 \\
0.55\end{array}$ & $\begin{array}{l}1.10 \\
0.99\end{array}$ & $\begin{array}{l}1.50 \\
1.45\end{array}$ & $\begin{array}{l}0.14 \\
0.25\end{array}$ \\
\hline $\begin{array}{l}\text { Toyo } \\
\text { Atomized }\end{array}$ & $\begin{array}{l}10 \\
20^{*}\end{array}$ & $\begin{array}{l}0.30 \\
0.27\end{array}$ & $\begin{array}{l}0.68 \\
0.55\end{array}$ & $\begin{array}{l}1.17 \\
0.98\end{array}$ & $\begin{array}{l}0.13 \\
0\end{array}$ \\
\hline $\begin{array}{l}\text { Kerr } \\
\text { Spheraloy }\end{array}$ & $\begin{array}{l}20^{*} \\
30\end{array}$ & $\begin{array}{l}0.41 \\
0.29\end{array}$ & $\begin{array}{l}0.85 \\
0.59\end{array}$ & $\begin{array}{l}1.34 \\
0.84\end{array}$ & $\begin{array}{l}0.14 \\
0.06\end{array}$ \\
\hline $\begin{array}{l}\text { SS White** } \\
\text { New T.D. }\end{array}$ & $30^{*}$ & 0.34 & 0.59 & 0.93 & 0.06 \\
\hline
\end{tabular}

* Manufacturer's direction.

** Conventional alloy as control.

\subsection{Initial hardening time of mix}

Time of initial hardening of amalgam mixes before filling was measured by a new method and means of 5 measurements are given in Table 11 and Fig. 14. As stated earlier, the time thus measured means the time-point at which the amalgam mix was hard enough not to break down under the Gillmore needle with the alteration of loads.

A load $1(\times 454 \mathrm{~g}, 1$ pound) appearing in the figure is the same one as used in specification test for setting time of cements. When this is used as a basic yardstick, two G-C products are found to harden most rapidly after trituration, (S) and (T) begin hardening at a slow speed and, $(\mathrm{K})$ and $(\mathrm{C})$ are in the middle. 
TABle 11

Initial Hardening Time of Mixes

\begin{tabular}{|c|c|c|c|c|c|}
\hline \multirow{3}{*}{ Amalgam } & \multirow{3}{*}{$\begin{array}{c}\text { Time of Mixing* } \\
\text { (Sec.) }\end{array}$} & \multicolumn{4}{|c|}{ Load ( X 454g) } \\
\hline & & $1 / 4$ & $1 / 2$ & 1 & 2 \\
\hline & & \multicolumn{4}{|c|}{ Time of Initial Hardening (Sec.) } \\
\hline $\begin{array}{l}\text { Shofu } \\
\text { Spherical }\end{array}$ & 20 & 75 & 100 & 155 & 210 \\
\hline $\begin{array}{l}\text { G-C } \\
\text { Atomic }\end{array}$ & 5 & 30 & 50 & 70 & 120 \\
\hline $\begin{array}{l}\text { G-C } \\
\text { Atomic Pellet }\end{array}$ & 8 & 40 & 50 & 70 & 110 \\
\hline $\begin{array}{l}\text { Toyo } \\
\text { Atomized }\end{array}$ & 20 & 80 & 115 & 165 & 230 \\
\hline $\begin{array}{l}\text { Kerr } \\
\text { Spheraloy }\end{array}$ & 20 & 55 & 75 & 120 & 175 \\
\hline $\begin{array}{l}\text { SS White** } \\
\text { New T.D. }\end{array}$ & 30 & 55 & 65 & 135 & 255 \\
\hline
\end{tabular}

* Manufacturer's direction

** Conventional alloy as control.

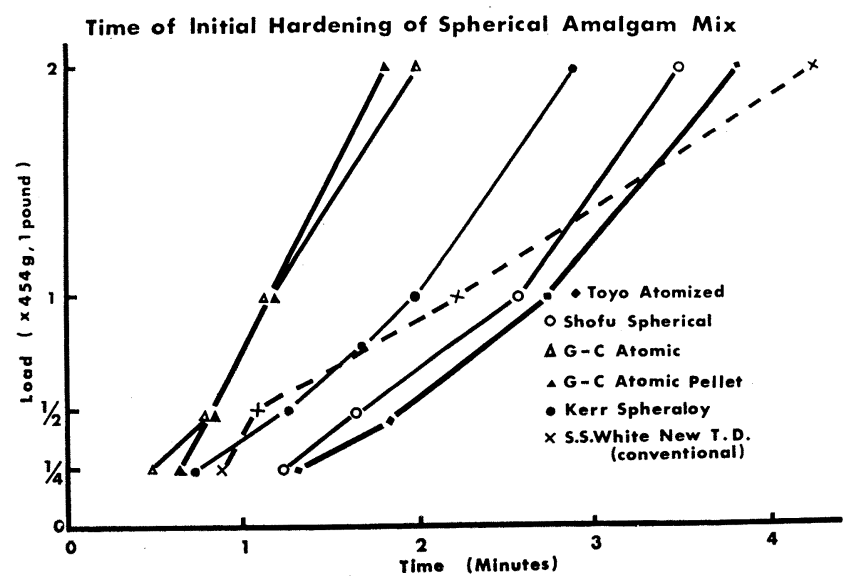

Fig. 14

\section{Discussion}

Most of the literature on dental amalgam is agreed that a restoration is attended by good results when the amount of residual mercury contained in it is small. It is already established[7] that, as compared with conventional alloy, spherical alloy can be readily triturated with less than one-half mercury and, for this reason, the amount of residual mercury is correspondingly smaller. 
As is implied by the name, the original spherical shape retains in the fully set amalgam, amount of reaction between the spherical particles and mercury is much smaller[4]. Moreover, spherical amalgam has such advantages as excellent pre-setting flow or plasticity and, in consequence, more compact restoration having less porosity. It is to be emphasized that the mechanical properties of spherical amalgam, condensed under a low condensation pressure in particular, are found much better than those of conventional amalgam[7].

Of various test items, differences of properties among individual alloys were more or less similar in findings concerning their compressive, tensile and marginal strengths.

Clinical consideration of the required strength of amalgam restoration after final set is irrelevant to the present study and it is pointed out here that difference in the initial strength before or during setting will have a serious clinical implication.

As given in Table 5 and Fig. 8, 3 different strengths are compared with the value of (C) as 100, after the time elapse of 5 hours. Of these, (GA) is found to be particular low, with the tensile strength being as low as $50 \%$. The marginal strength of this alloy corresponds to about $75 \%$ of (C). Since patients usually begin to eat after about 3-5 hours from their treatment, reduction in these strengths at this time-point will create a clinical problem.

Flow gives similar results to those of strengths except $(\mathrm{K})$ has the largest degree of flow contrary to the compressive strength is highest. It has been maintained that a sufficiently strong amalgam has a small flow[18]. But as the setting reaction and structure of spherical amalgam greatly differ from that of conventional alloy, we hold that spherical amalgam should be considered in an entirely different light.

(GA) and (GP) are observed to increase their flow in proportion to the length of trituration time, whereas $(\mathrm{S})$ and $(\mathrm{T})$ are less affected in this respect. Flow of all the amalgams, however, comes within the range of tolerance provided for in the specification. In view of rapid advance and renovation in the dental materials, the range of current specification needs to be carefully reexamined for possible amendment.

Corrosive tendency in an artificial saliva as determined by the polarography is in keeping with the mechanical properties. On the whole, a strong amalgam is known to be correspondingly corrosion-resistant[16]. This is explained by the fact that $\gamma_{2}$ phase in the fully set amalgam structure is most susceptible to a corrosion as this phase is the weakest. In these properties, two products (GA) and (GP) are inferior to other products and also to $(\mathrm{C})$.

Unshakable advantage of spherical amalgam over conventional amalgam is demonstrated in the fact that the former is able to assume a far better initial strength packed by a relatively low condensation pressure[1, 5, 6, 7,]. A short trituration time may be one of the reasons that two G-C products are inferior in respect of their mechanical properties. The instructed trituration time of 5 to 8 seconds may be necessary for the attainment of a slight setting expansion and, if so, other mechanical properties are sacrificed because of insufficient trituration.

Major difference between the dimensional changes of conventional and spherical amalgams is that there is no initial contraction in the latter when measured 15 minutes after the start of trituration[7,9]. As revealed in our tests, curves of dimensional change 
varied from product to product. Two G-C products start to expand and attain to a fixed level after 6 hours. (S) and (T) give an expansion initially and contract a little afterward, resembling the behavior of conventional amalgam. $(\mathrm{K})$ continues a contraction after 6 hours, contrary to the case of (GA). Differences in these curves are due to differences of the reactions, different particle sizes, their shape and distribution, manufacturing processes etc. Because of shorter trituration time, (GA) and (GP) give an expansion. Although (S) and (T) slightly contract when triturated for a length of time as instructed, they expand when the length of trituration is reduced to 5 seconds as is the case with (GA). It is not logical, however, to obtain a slight expansion at the cost of other important properties.

It should be borne in mind that the size and shape of test specimen as given in the current specification test are quite different from those of an actual restoration and the conditions under which a test specimen is placed do not exactly correspond to the actual oral cavity where the restoration is surrounded by the tooth. There seems no scientific justification for the current provision that there should be an expansion of 0-20 $\mu / \mathrm{cm}$, nor is there any clinical necessity for this expansion. Even if an amalgam is prepared with the specified expansion value, some of interstice is bound to be noted between the restoration and tooth[19, 20, 21]. In terms of clinical observation, there seems to be no appreciable difference between contracting amalgam and expanding amalgam[22]. From these considerations, we maintain that amalgam should be selected on the basis of as little dimensional change as possible without any regard to its expansion or contraction.

As a new approach to our amalgam studies, we paid attention to the initial hardening time of an amalgam mix after it has been triturated. This is closely linked to the ease or difficulty of handling an amalgam mix into a prepared cavity following the trituration. After various attempts, we came to devise a method by which it is possible to determine the time of hardening of an amalgam mix by applying Gillmore needle on it. Time was measured until the needle fails to penetrate into the ball of mix or fails to break the ball. Table 10 and Fig. 14 compare these initial hardening times of amalgam mixes prepared under the instructed trituration time and alloymercury ratio respectively.

When a load of $454 \mathrm{~g}$ (one pound) is taken to be a standard, (K) is somewhat faster and (S) and (T) are slower as against 2 minutes 15 seconds of conventional amalgam. Two G-C products, (GA) and (GP), harden much faster than the rest.

By the use of this chart, it is possible to know a particular hardening at a given time-point by referring to a load involved. More than 1 minute is spent in the majority of dentists of Japan before a filling procudure is attempted after an amalgam has been triturated[23]. In terms of this practice, $(\mathrm{S}),(\mathrm{T}),(\mathrm{C})$ and $(\mathrm{K})$ are easy of manipulation while two G-C products are difficult to handle.

The hardness of amalgam mix before being filled into prepared cavity has not been scientifically studied before. We trust that our method here described will be eventually adopted as part of specification testing in the future. More details of this property will be investigated. 


\section{Conclusions}

Our comparative study of 5 spherical amalgams commercially available today with a conventional amalgam in various properties brought to light the following conclusions.

1) Differences in the properties were noted even among spherical alloys.

2) Although some spherical alloys gave an expansion in much shorter trituration time than others, this was done at the cost of other physical properties. With these products, particular attention was directed to the reduction of initial strength which is clinically important.

3) Certain spherical alloys had similar properties and remained more or less unaffected by changes in conditions. These products were found to be the best of all amalgams tested.

4) As an approach to determine the ease or difficulty of handling before it is filled in a prepared cavity, a new method was devised to establish the initial hardening time of amalgam mix. Dentist will be greatly assisted to judge the ease or difficulty of handling amalgam mix by the information from this test.

\section{References}

[1] Demaree, N. C. and Taylor, D. F.: Properties of Dental Amalgams made from Spherical Alloy Particles, J. Dent. Res. 41: 890, 1962.

[2] Parsons, D. L. and Taylor, D. F.: Effect of Composition and Particle Size on the Mechanical Properties of Dental Amalgam made from Spherical Alloys, presented at the I. A. D. R. annual meeting for 1967.

[3] Taylor, D. F. and Burnham, J. B.: Characteristics of Spherical-Alloy Amalgams of known Particle Size Distribution, presented at the I. A. D. R. annual meeting for 1967.

[4] Wing, G. and Ryge, G.: Setting Reactions of Spherical-Particle Amalgams, J. Dent. Res. 44: 1325, 1965.

[5] Koran, A. and Asgar, K.: A Comparison of Dental amalgams made from a Spherical Alloy and from a Comminuted Alloy, J. A. D. A., 75: 912, 1967.

[6] Eden, G. T. and Waterstrat, R. M.: Effect of Packing Pressures on the Properties of Spherical Alloy Amalgams, J. A. D. A. 74: 1024, 1967.

[7] Nagai, K., Ohashi, M. and Miyatsu, H.: Studies on Spherical Amalgam Alloy in the Light of Dental Technology, J. Nihon Univ. Sch. Dent. 8: 149, 1966.

[8] Nagai, K., Ohashi, M. and Hasegawa, K.: Comparative Study on the Marginal Strengths of Conventional and Spherical Amalgam Alloys, J. Nihon Univ. Sch. Dent. 9: 49, 1967.

[9] NaGAI, K. and OHASHI, M.: Studies on the Dimensional Change of Dental Amalgams with Reference to the Dimensional Change of Spherical Amalgam developed in Japan-, J. Nihon Univ. Sch. Dent., 9: 1, 1967.

[10] NagaI, K. and OHashi, M.: Three Studies on a newly developed Spherical Amalgam in Japan and Suggestions for its Specification, J. Nihon Univ. Sch. Dent., 9: 43, 1967. 
[11] Nagai, K. and OHASHI, M.: Study on the Corrosion Resistance of Spherical Amalgam with Reference to the Human Saliva, J. Nihon Univ. Sch. Dent., 9:143, 1967.

[12] Plump, E. H.: Spherical Alloy Amalgam: A Clinical Study, presented at the I. A. D. R. annual meeting for 1967.

[13] Nagai, K., Ohashi, M., Habu, H. and Nemoto, K.: The Analysis of Pressure exerted in the Condensation of Amalgam, (Unpublished paper).

[14] ASTM C496-66T.

[15] Tsurumaki, K.: The Determination of Corrosions of Dental Alloys by the Use of Polarograph, Shika-Geppo, 34: 73, 1960.

[16] Habu, H.: A Polarographic Study on the Corrosion Tendency of Dental Amalgam, J. Nihon Univ. Sch. Dent., 6:6, 1964.

[17] Federation Dentaire Internationale: Specification for Alloy for Dental Amalgam, International Dental Journal, 9:421, 1959.

[18] Peyton, F. A., others.: Restorative Dental Materials, Second edition, The C. V. Mosby Co., Saint Louis, 1964.

[19] Nelson, R. J., Wolcott, R. B. and Paffenbarger, G. C.: Fluid Exchange at the Margins of Dental Restorations, J. A. D. A., 44: 288, 1952.

[20] GoInG, R. E. and MASSler, M.: Influence of Cavity Liners under Amalgam Restorations on Penetration by Redioactive Isotopes, J. Pros. Den., 11: 298, 1961.

[21] Christen, A. G., and Mitchell, D. F.: A Fluorescent Dye Method for Demonstrating Leakage around Dental Restorations, J. Dent. Res., 45: 1485, 1966.

[22] McDonald, R. E. and Phillips, R. W.: Clinical Observation on a Contracting Amalgam Alloy, J. Dent. Res. 45: 1485, 1950.

[23] Nagai, K., Ohashi, M. and Habu, H.: Clinical Observations of Various Time Procedures required in Amalgam Manipulation, (Unpublished paper). 